\title{
A 57-year-old man with rapidly progressive pulmonary hypertension
}

\author{
Federico Raimondi ${ }^{1,2}$, Caterina Conti ${ }^{1}$, Luca Novelli ${ }^{1}$, Francesco Tarantini ${ }^{1}$, Giuseppe Ciaravino ${ }^{1}$, \\ Piermario Scuri $^{3}$, Aurelia Grosu ${ }^{3}$, Daniela Chinaglia ${ }^{4}$, Lorenzo SC Grazioli ${ }^{5}$, Andrea Gianatti ${ }^{4}$, \\ Ferdinando L. Lorini ${ }^{5}$, Michele Senni ${ }^{3}$, Fabiano Di Marco ${ }^{1,2}$ \\ ${ }^{1}$ Pulmonary Medicine Unit, ASST Papa Giovanni XXIII Hospital, Bergamo; ${ }^{2}$ University of Milan; ${ }^{3}$ Cardiology Unit, \\ ASST Papa Giovanni XXIII Hospital, Bergamo; ${ }^{4}$ Department of Pathology, ASST Papa Giovanni XXIII Hospital, \\ Bergamo; ${ }^{5}$ Anesthesiology Intensive Care Unit 3, ASST Papa Giovanni XXIII Hospital, Bergamo, Italy
}

\begin{abstract}
Pulmonary tumor thrombotic microangiopathy (PTTM) is a rare condition associated with neoplastic disorders, predominantly gastric cancer, leading to pre-capillary pulmonary hypertension (PH). The pathologic mechanism involved is a fibrocellular intimal proliferation of small pulmonary vessels sustained by nests of carcinomatous cells lodged in pulmonary vasculature. Clinical presentation is nonspecific, including progressive dyspnea and dry cough. Diagnosis of PTTM is extremely challenging ante-mortem
\end{abstract}

Correspondence: Caterina Conti, Pulmonary Medicine Unit, ASST Papa Giovanni XXIII Hospital, Piazza OMS 1, Bergamo, Italy. Tel. + 39.035.2673445 - Fax: +39.035.2674889.

E-mail: cconti@asst-pg23.it

Key words: Pulmonary hypertension; dyspnea; microangiopathy.

Authors Contributions: FR, CC, LN, FDM, responsible for manuscript drafting and revision; FT, GC, PS, AG, MS, LSCG, FLL revised the manuscript and were primary involved in patient care and management; DC, AG, obtained histopathological images and supported other physicians in the analysis. All the authors provided a substantive intellectual contribution, read and approved the final version of the manuscript and agreed to be accountable for all aspects of the work.

Conflict of interest: None of the authors have any financial interests which would create a conflict of interest.

Ethics approval and consent to participate: No Ethical Committee approval was required for this case report, because this article does not contain any studies with human participants or animals.

Consent for publication: A written consent for publication was obtained from the patient's spouse.

Received for publication: 15 May 2021.

Accepted for publication: 13 July 2021.

${ }^{\circ}$ Copyright: the Author(s), 2021

Licensee PAGEPress, Italy

Monaldi Archives for Chest Disease 2022; $92: 1930$

doi: 10.4081/monaldi.2021.1930

This article is distributed under the terms of the Creative Commons Attribution Noncommercial License (by-nc 4.0) which permits any noncommercial use, distribution, and reproduction in any medium, provided the original author(s) and source are credited. and prognosis is poor. Here we describe the case of a middle-aged man, without known previous cancer history. The clinical course was rapidly unfavorable, with progressive dyspnea and $\mathrm{PH}$ associated with hemodynamic instability, eventually culminating in patient's death. PTTM diagnosis was made post-mortem. PTTM should be considered in any patient presenting with unexplained $\mathrm{PH}$, especially if it is rapidly progressive, poorly responsive to standard approaches or there is suspected history of malignancy. A prompt diagnosis of PTTM could help in bringing light into this still under-recognized condition.

\section{Introduction}

Pulmonary hypertension $(\mathrm{PH})$ is a progressive disease which often leads to premature death. Although the severity of this clinical entity, the diagnosis is often delayed because the presenting features of $\mathrm{PH}$ are frequently attributed erroneously to age, deconditioning, or a coexisting medical condition. The most updated clinical classification of $\mathrm{PH}$ in adults was recently presented in the $6^{\text {th }}$ World Symposium on Pulmonary Hypertension (2019) [1]. Amongst the multitude of etiologies leading to $\mathrm{PH}$ development, some tumor-related forms of $\mathrm{PH}$ have been described, including tumoral pulmonary microvascular conditions, such as Pulmonary Tumor Thrombotic Microangiopathy (PTTM).

\section{Case Report}

A 57-year-old man was referred to our hospital (Papa Giovanni XXIII, Bergamo, Italy) after six weeks history of progressive dyspnea and dry cough. He also complained loss of appetite, epigastric pain after eating and a seven kilograms weight loss in few months. He was a heavy smoker (40 pack-years) and led a stressful life as a betting center director. He was married, denied substance abuse and only drank alcohol with moderation. His medical history was unremarkable except for legs varicose veins and a previous cutaneous lipoma excision. The arterial blood gas analysis in room air at presentation showed respiratory alkalosis with a $\mathrm{pH}$ of 7.52 , a partial arterial pressure of carbon dioxide $\left(\mathrm{PaCO}_{2}\right)$ of $26 \mathrm{mmHg}$ and a partial arterial pressure of oxygen $\left(\mathrm{PaO}_{2}\right)$ of $65 \mathrm{mmHg}$. On admission he was normotensive and tachycardic (blood pressure $120 / 65 \mathrm{mmHg}$ and 115 beats/min, respectively), with a respiratory rate of 26 breaths $/ \mathrm{min}$. He was afebrile without any signs of systemic or respiratory infection. The chest examination was unre- 
markable and murmurs were not detectable at the heart auscultation. The abdomen was tender in epigastric region without masses or organomegaly. No peripheral edema or signs of deep venous thrombosis were observed at lower limbs.

The patient underwent a computed tomography pulmonary angiography (CTPA), showing mediastinal and bilateral hilar lymphadenopathy, a pulmonary nodule of $11 \mathrm{~mm}$ in the middle lobe and diffuse centrilobular ground glass opacities with interlobular septum thickening, while pulmonary embolism was ruled out (Figure 1A). A transthoracic echocardiogram (TTE) showed a $10 \mathrm{~mm}$ pericardial effusion and an estimated systolic pulmonary pressure (PAPs) of 70 $\mathrm{mmHg}$, a mild reduction of right ventricle (RV) systolic function associated with a tricuspid annular plane systolic excursion (TAPSE) of $16 \mathrm{~mm}$ (normal value $\geq 18 \mathrm{~mm}$ ) and a tricuspid regurgitation velocity of $3.87 \mathrm{~m} / \mathrm{s}$ (normal value $<2.8 \mathrm{~m} / \mathrm{s}$ ). Blood biochemical tests were unremarkable for inflammation markers, autoimmune dis- eases and human immunodeficiency virus (HIV). Brain natriuretic peptide (BNP) values were elevated (311 ng/l, with a normal value $<100 \mathrm{ng} / \mathrm{l})$. Soon after the initiation of diuretics, which led to a good clinical and biochemical response, the patient underwent a 6-minute walking test showing significant desaturation (420 meters walking distance, $\mathrm{SpO}_{2}$ at rest 98\%, nadir $\mathrm{SpO}_{2}: 93 \%$ ).

A TTE was repeated few days later since the patient experienced hemodynamic deterioration, with worsening dyspnea and syncopal episodes during cough. It showed a worsening of indirect signs of pulmonary hypertension (PH), with an estimated PAPs of $100 \mathrm{mmHg}$ and paradoxical septal motion. Right heart catheterization (RHC) confirmed the presence of a severe pre-capillary $\mathrm{PH}$ with a reduced cardiac index (systolic, diastolic, and mean pulmonary artery pressure: $80 / 30 / 47 \mathrm{mmHg}$, respectively; right atrium pressure of $3 \mathrm{mmHg}$; wedge pressure of $5 \mathrm{mmHg}$; cardiac index $1.59 \mathrm{~L} / \mathrm{min} / \mathrm{m}^{2}$, pulmonary vascular resistance $15 \mathrm{WU}$ ).
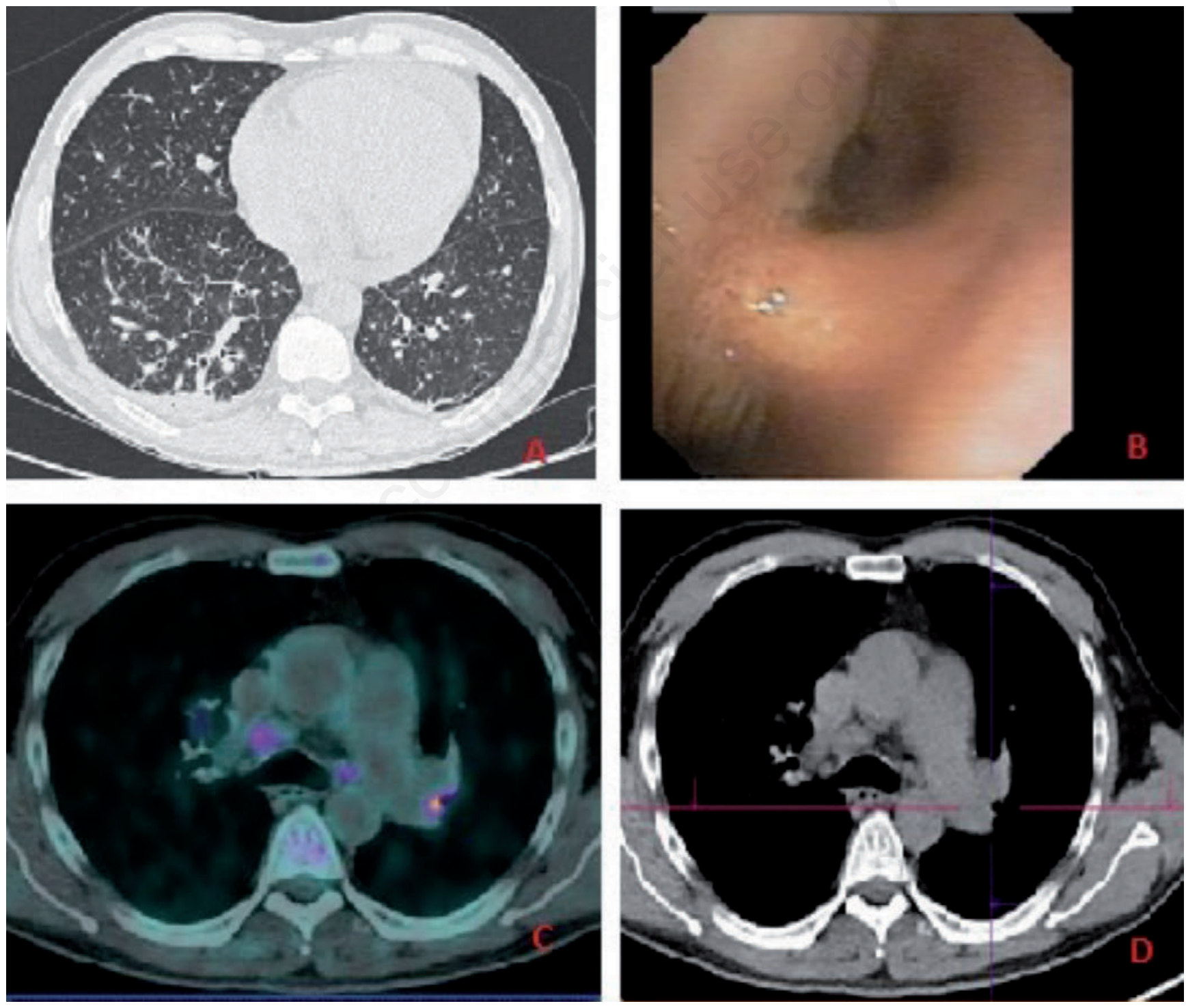

Figure 1. A) Computed tomography pulmonary angiography showing a pulmonary nodule of $11 \mathrm{~mm}$ in the middle lobe and centrilobular ground glass opacities with interlobular septum thickening. B) Fiberoptic bronchoscopic image showing an enlarged right secondary carina without mucosal abnormalities. C,D) 18F-FDG PET/CT scan showing significantly increased radiotracer uptake (SUV 10.1) within hilar and mediastinal lymphnodes. 
Abdominal and lower limb ultrasonography were unremarkable while coronary angiography showed a significant stenosis at the middle segment of right coronary. A ${ }^{18} \mathrm{~F}-\mathrm{FDG}$ PET/CT scan was then obtained showing significantly increased radiotracer uptake (Standardized Uptake Value, SUV, max 10.1) within hilar and mediastinal lymphnodes (Figure 1 C,D). No other significant uptakes were reported. Looking for a possible neoplastic disease, the patient underwent a bronchoscopy with bronchoalveolar lavage (BAL) showing an enlarged right secondary carina without mucosal abnormalities or other endoscopic detectable lesions (Figure 1B). No malignant cells were detected on BAL. A transbronchial needle aspiration (TBNA) of the right carina was not feasible because the patient became hemodynamically unstable.

Considering the RHC results, consistent with a pre-capillary form of $\mathrm{PH}$ and the negativity of all the investigations performed, a Group 1 PH was initially hypothesized and the patient started a treatment with sildenafil $20 \mathrm{mg}$ tris in die associated with dobutamine and loop diuretics without any benefit in terms of hemody- namic status but with worsening hypotension that led to the necessity of moving the patient to intensive care unit (ICU). After multidisciplinary evaluation and considering the lymph nodes uptake at ${ }^{18} \mathrm{~F}$-FDG PET/CT scan with enlarged right carina pointing to a possible neoplastic underlying disease, an endobronchial ultrasound (EBUS)-TBNA of hilar and mediastinal lymphnodes was attempted with the assistance of the extracorporeal life support (ECLS) team alerted for the high-risk procedure. A sudden cardiac arrest occurred when the bronchoscope passed the vocal cords with prompt starting of ECLS maneuvers. Unfortunately, given the evidence of severe brain damage and the absence of available treatments for the unknown condition of the patient, palliative care was provided and the patient died shortly after.

The autopsy report identified the presence of a gastric signetring cell carcinoma (WHO 2010) of the gastric corpus and antrum (diffuse type sec. Lauren). Massive pulmonary thromboembolism was found, with almost the totality of small and medium pulmonary arteries involved (Figure 2). The occlusive thrombosis,

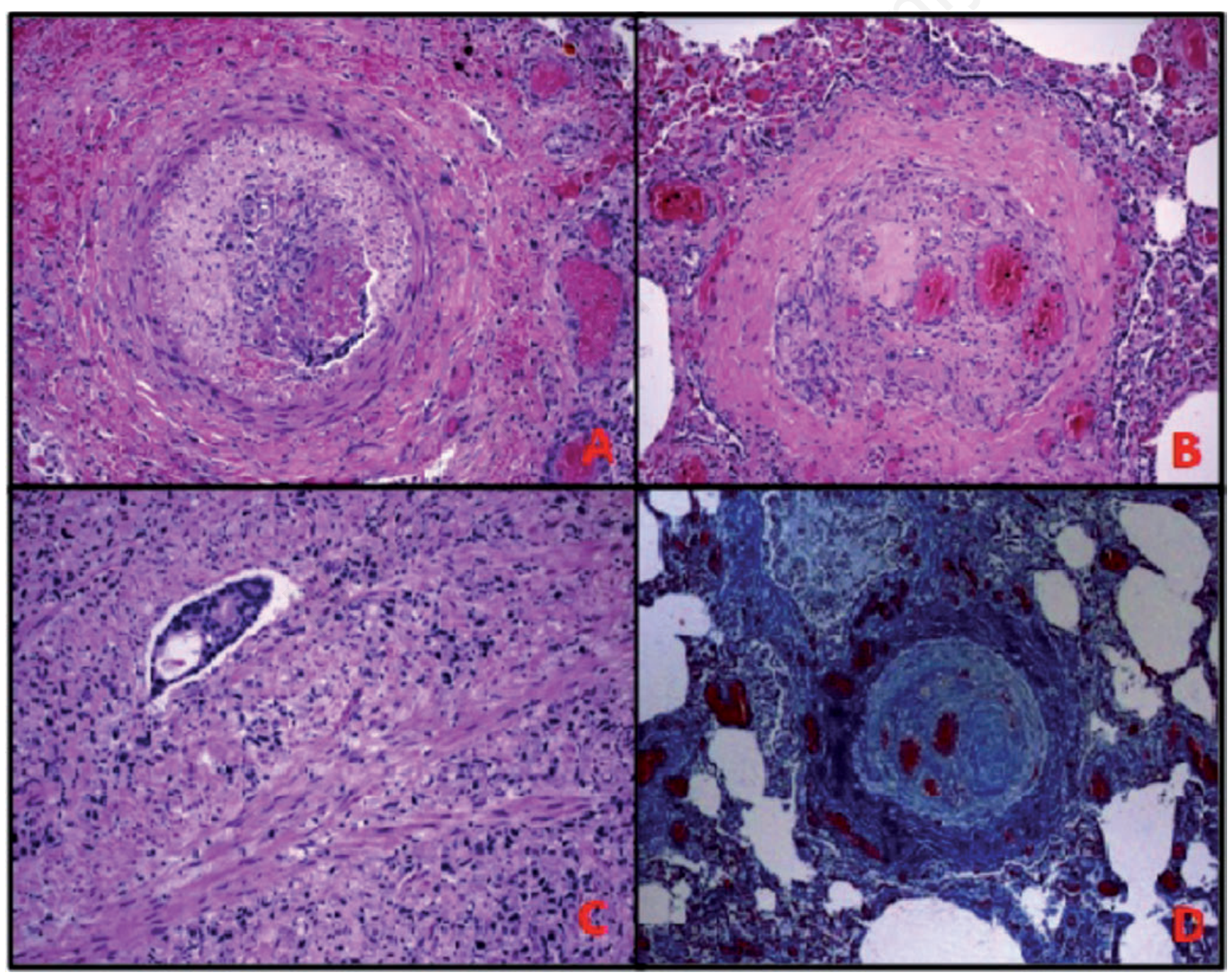

Figure 2. A) Pulmonary arterial vascular structure occluded by fibrinous thrombus, neoplastic embolus and concentric fibrosis with intimal proliferation; H\&E staining, 10x magnification. B) Pulmonary arterial vessel occluded by fibrosis, intimal proliferation; associated eccentric revascularization; H\&E staining, 10x magnification. C) Gastric wall with signet ring cells carcinoma and neoplastic invasion of lymphatic vessels; H\&E staining, 10x magnification. D) Masson trichromic stain, 5x magnification; light blue, endoluminal arterial fibrosis; blue, extensive perivascular fibrosis; red, vessel recanalization. 
partially recanalized, was both hematic and neoplastic with endoluminal aspects of fibrosis. Moreover, a massive neoplastic involvement of liver, pancreas, lungs, kidneys and meningeal vessels was described. The autopsy findings are conclusive for pulmonary tumor thrombotic microangiopathy (PTTM).

\section{Discussion}

PTTM is a rare process associated with neoplastic diseases in which malignant cells migrate to the pulmonary vasculature [2]. The interaction between neoplastic and endothelial cells initiates clot formation, leading to the release of cytokines including vascular endothelial growth factor (VEGF) and platelet-derived growth factor (PDGF). This process triggers macrophage recruitment and intimal proliferation [3-5]. The vessel remodeling leads to an increase in pulmonary artery pressures and consequent right heart failure, often within weeks from the symptoms' onset. Therefore, PTTM is not a simple occlusion of small pulmonary arteries by metastatic tumor cells (i.e., pulmonary tumor embolism), but a profound remodeling of pulmonary vessels culminating in an occlusive fibrocellular intimal proliferation. PTTM is most commonly associated with gastric adenocarcinoma but has been described with increasing frequency in the last few years in numerous other neoplastic conditions such as hepatocarcinoma, breast cancer, lung adenocarcinoma, urothelial and ovarian carcinoma [3,6-11]. Indeed, typical histological features of PTTM were reported in the $16.7 \%$ of autopsy cases of gastric adenocarcinoma while in other carcinomas ranged from $1.4 \%$ to $3.3 \%[2,3,12]$.

PTTM symptoms pattern is nonspecific, including worsening dry cough, possibly due to neoplastic infiltration of airway mucosa with stimulation of cough receptors and rapidly progressive dyspnea or orthopnea [5]. These last symptoms might be amenable to an increased RV afterload, reduced RV cardiac output, and hypoxemia, moreover airflow obstruction triggered by cough could be a further mechanism involved. Other symptoms may be related to the site of the primary malignancy. Hypoxemia can be explained by reduction in RV and left ventricle (LV) cardiac output due to severe $\mathrm{PH}$, increased shunting, tumor infiltration of the alveoli and impairment of gas diffusion across the capillary membrane. It represents the most relevant sign presented by the patients affected by PTTM and is a poor prognostic factor with frequent relentless progression towards death. The most common laboratory abnormalities include elevation of D-dimer and of LDH, the presence of anemia and thrombocytopenia and, possibly, signs of disseminated intravascular coagulopathy (DIC) or tumor cell infiltration of the bone marrow [12]. Nevertheless, it is possible that a patient with underlying PTTM presents without any of these abnormalities. Radiological tests include the chest CT scan, that commonly shows ground glass opacities, centrilobular nodules, septal thickening, mediastinal/hilar lymphadenopathies, and consolidations [5]. All those abnormalities probably result from the hematogenous spread of malignancy through pulmonary arterioles, engorgement of lymphatic channels and drainage of malignant cells via the lymphatics or the thoracic duct in gastric cancer. The use of ${ }^{18} \mathrm{~F}-\mathrm{FDG}$ PET/CT scan for the detection of primary malignancies causing PTTM has a main limitation due to the reduced tracer avidity of some types of adenocarcinomas, resulting in false negative tests. TTE is a useful screening tool to identify PH and to quantify the degree of RV dysfunction.

In the ERS/ATS Guidelines, PTTM is classified under the Group $5 \mathrm{PH}$ category [13]. RHC is indicated to assess pulmonary hemodynamic and to guide initiation of diuretics, inotropic support and to consider off label PH therapy. Furthermore, it allows the sampling of blood from the pulmonary artery, which in some cases reveals the presence of cells from the primary malignancy. Nevertheless, the rapid clinical decline often hampers its performance.

Several treatments have been attempted for patients with PTTM. Case reports documented a reduction of mean pulmonary artery pressure (mPAP) with the use of imatinib, sildenafil, ambrisentan, total gastrectomy, bevacizumab and TS- 1 chemotherapy for the primitive cancer [14-17]. However, the matter is particularly challenging because the average time from symptoms onset to death is approximately 9.5 weeks, with difficult ante-mortem diagnosis and consequent treatment [5]. Almost invariably the outcome is unfavorable and the vast majority of cases are diagnosed post-mortem. When obtained, lung histology reveals nests of tumor cells within the lumen of pulmonary pre-capillary vessels, with surrounding fibrin deposition along with fibrocellular intimal hyperplasia.

\section{Conclusions}

PTTM should be considered in any patient presenting with unexplained $\mathrm{PH}$, especially if it is rapidly progressive, poorly responsive to standard approaches or there is suspected history of malignancy. A prompt diagnosis of PTTM could help in bringing light into this still under-recognized condition and it could allow the identification and treatment of the primitive cancer.

\section{References}

1. Galiè N, McLaughlin V V., Rubin LJ, Simonneau G. An overview of the 6th World Symposium on Pulmonary Hypertension. Eur Respir J 2019;53:1802148.

2. Von Herbay A, Illes A, Waldherr R, Otto HF. Pulmonary tumor thrombotic microangiopathy with pulmonary hypertension. Cancer 1990;66:587-92.

3. Chinen K, Tokuda Y, Fujiwara M, Fujioka Y. Pulmonary tumor thrombotic microangiopathy in patients with gastric carcinoma: An analysis of 6 autopsy cases and review of the literature. Pathol Res Pract 2010;206:682-9.

4. Price LC, Wells AU, Wort SJ. Pulmonary tumour thrombotic microangiopathy. Curr Opin Pulm Med 2016;22:421-8.

5. Godbole RH, Saggar R, Kamangar N. Pulmonary tumor thrombotic microangiopathy: a systematic review. Pulm Circ 2019;9:2045894019851000.

6. Okubo Y, Wakayama M, Kitahara K, et al. Pulmonary tumor thrombotic microangiopathy induced by gastric carcinoma: Morphometric and immunohistochemical analysis of six autopsy cases. Diagn Pathol 2011;6;27.

7. Uga S, Ikeda S, Matsukage SI, Hamada M. An autopsy case of acute cor pulmonale and paradoxical systemic embolism due to tumour cell microemboli in a patient with breast cancer. Case Reports 2012;2012:bcr2012006682.

8. Buser M, Felizeter-Kessler M, Lenggenhager D, Maeder MT. Rapidly progressive pulmonary hypertension in a patient with pulmonary tumor thrombotic microangiopathy. Am J Respir Crit Care Med 2015;191:711-2.

9. Gru AA, Pai RK, Roma AA. Pulmonary tumor thrombotic microangiopathy in patients with low-grade ovarian serous neoplasm: A clinicopathologic review of 2 cases of a previous- 
ly unknown association. Int J Gynecol Pathol 2012;31:438-42.

10. Tanaka K, Nakasya A, Miyazaki M, et al. A case of hepatocellular carcinoma with respiratory failure caused by widespread tumor microemboli. Hukuoka Acta Medica 2011;102:298-302.

11. Malani AK, Gupta C, Kutty AVP, Betlej T. Pulmonary tumor thrombotic microangiopathy from metastatic gallbladder carcinoma: An unusual cause of severe pulmonary hypertension. Digest Dis Sci 2007;52:555-7.

12. Uruga H, Fujii T, Kurosaki A, et al. Pulmonary tumor thrombotic microangiopathy: A clinical analysis of 30 autopsy cases. Internal Med 2013;52:1317-23.

13. Galiè N, Humbert M, Vachiery JL, et al. 2015 ESC/ERS Guidelines for the diagnosis and treatment of pulmonary hypertension. Eur Respir J 2015;46:903-75.
14. Minatsuki S, Miura I, Yao A, et al. Platelet-derived growth factor receptor-tyrosine kinase inhibitor, imatinib, is effective for treating pulmonary hypertension induced by pulmonary tumor thrombotic microangiopathy. Int Heart J 2015;56:245-8.

15. Ogawa A, Yamadori I, Matsubara O, Matsubara H. Pulmonary tumor thrombotic microangiopathy with circulatory failure treated with imatinib. Internal Med 2013;52:1927-30.

16. Kayatani H, Matsuo K, Ueda Y, et al. Pulmonary tumor thrombotic microangiopathy diagnosed antemortem and treated with combination chemotherapy. Internal Med 2012; 51:2767-70.

17. Higo K, Kubota K, Takeda A, et al. Successful antemortem diagnosis and treatment of pulmonary tumor thrombotic microangiopathy. Internal Med 2014;53:2595-9. 